\title{
Combined Effect of Famine Exposure and Obesity Parameters on Hypertension in the Midaged and Older Adult: A Population- Based Cross-Sectional Study
}

\author{
Lin Zhang $\mathbb{D}^{1}{ }^{1}$ Liu Yang, ${ }^{1}$ Congzhi Wang, ${ }^{1}$ Ting Yuan, ${ }^{2}$ Dongmei Zhang, ${ }^{3}$ Huanhuan Wei, \\ Jing Li, ${ }^{4}$ Yunxiao Lei, ${ }^{2}$ Lu Sun, ${ }^{5}$ Xiaoping $\mathrm{Li}^{5}{ }^{5}$ Ying Hua, ${ }^{6}$ Hengying Che ${ }^{(D)},{ }^{7}$ \\ and Yuanzhen $\mathrm{Li} \mathbb{1 D}^{5}$ \\ ${ }^{1}$ Department of Internal Medicine Nursing, School of Nursing, Wannan Medical College, 22 Wenchang West Road, \\ Higher Education Park, Wuhu City, Anhui Province, China \\ ${ }^{2}$ Obstetrics and Gynecology Nursing, School of Nursing, Wannan Medical College, 22 Wenchang West Road, \\ Higher Education Park, Wuhu City, Anhui Province, China \\ ${ }^{3}$ Department of Pediatric Nursing, School of Nursing, Wannan Medical College, 22 Wenchang West Road, Higher Education Park, \\ Wuhu City, Anhui Province, China \\ ${ }^{4}$ Department of Surgical Nursing, School of Nursing, Wannan Medical College, 22 Wenchang West Road, Higher Education Park, \\ Wuhu City, Anhui Province, China \\ ${ }^{5}$ Department of Emergency and Critical Care Nursing, School of Nursing, Wannan Medical College, 22 Wenchang West Road, \\ Higher Education Park, Wuhu City, Anhui Province, China \\ ${ }^{6}$ Rehabilitation Nursing, School of Nursing, Wannan Medical College, 22 Wenchang West Road, Higher Education Park, \\ Wuhu City, Anhui Province, China \\ ${ }^{7}$ Department of Nursing, Yijishan Hospital, The First Affiliated Hospital of Wannan Medical College, Zheshan West Road, \\ Yijishan District, Wuhu City, Anhui Province, China
}

Correspondence should be addressed to Hengying Che; 2722659079@qq.com and Yuanzhen Li; yuanzhen.li@wnmc.edu.cn

Received 17 May 2021; Accepted 7 September 2021; Published 24 September 2021

Academic Editor: Sercan Erg $n$

Copyright (c) 2021 Lin Zhang et al. This is an open access article distributed under the Creative Commons Attribution License, which permits unrestricted use, distribution, and reproduction in any medium, provided the original work is properly cited.

Objectives. Undernutrition early in life may increase the incidence of adverse effects on adult health. The relations between undernutrition and obesity parameters (body mass index (BMI) and WC (waist circle)) and hypertension were often contradictory. Our study is aimed at identifying the combined effects of famine exposure and obesity parameters on hypertension in middle-aged and older Chinese. Design. A population-based cross-sectional study. Setting. Data were selected from the China Health and Retirement Longitudinal Study Wave2011 (CHARLS Wave2011). Participants. The sample included 12945 individuals aged 45 to 96. Main Outcome Measurements. The study analyzed data from 12945 middle-aged and older Chinese selected from CHARLS Wave2011. Differences between baseline characteristics and famine exposure/BMI levels/WC levels were evaluated using the $t$-, Chi-square- $\left(\chi^{2}-\right)$, and $F$-test. Then, the difference in the prevalence of hypertension between baseline characteristics was estimated by the $t$ - and $\chi^{2}$-test. Finally, multivariable-adjusted logistic regression models were used to explore the associations of famine exposure and obesity parameters with odds of prevalence of hypertension. Results. Among the 12945 participants, 1548 (11.96\%) participants had been exposed to the Chinese famine during the fetal group, whereas 5101 (39.41\%) participants and 4362 (33.70\%) participants had been exposed to the famine during childhood and adolescence/adult group, respectively. Regarding the participants with BMI levels, 3746 (28.94\%) were overweight, and $1465(11.32 \%)$ were obese, whereas $5345(41.29 \%)$ of the participants with WC levels were obese, respectively. Furthermore, 1920 (31.17\%) had hypertension in males and 2233 (32.91\%) in females. In multivariable-adjusted models, famine exposure and obesity parameters were related with prevalence of hypertension independently in total populations ((1) model three ${ }^{c}$, famine exposure with prevalence of hypertension: the fatal-exposed vs. no-exposed group (OR1.27; 95\% CI 1.08, 1.49); childhood-exposed vs. no-exposed group (OR1.64; 95\% CI 1.44, 1.87); the adolescence/adult-exposed vs. no-exposed 
group (OR3.06; 95\% CI 2.68, 3.50); $P$ for trend $<0.001$; (2) model three ${ }^{\mathrm{e}}$, famine exposure with prevalence of hypertension: the fatal-exposed vs. no-exposed group (OR1.25; 95\% CI 1.06, 1.47); childhood-exposed vs. no-exposed group (OR1.52; 95\% CI $1.34,1.73)$; the adolescence/adult-exposed vs. no-exposed group (OR2.66; 95\% CI 2.33, 3.03); $P$ for trend <0.001; (3) model three ${ }^{\mathrm{g}}$, BMI levels with prevalence of hypertension: overweight vs. normal (OR1.75; 95\% CI 1.60, 1.91); obesity vs. normal (OR2.79; 95\% CI 2.48, 3.15); $P$ for trend $<0.001$; (4) WC levels with prevalence of hypertension: overweight vs. normal (OR1.42; 95\% CI 1.36, 1.48)). When stratified by sex, results in both males and females were mostly similar to those in the total population. In general, interaction analysis in the multivariable-adjusted model, compared with the combination of normal BMI/WC levels and no-exposed famine group, all groups trended towards higher odds of prevalence of hypertension (the greatest increase in odds, adolescence/adult-exposed group with obesity in BMI levels: (OR8.13; 95\% CI 6.18, 10.71); adolescence/adult-exposed group with obesity in WC levels: (OR6.36; 95\% CI 5.22, 7.75); $P$ for interaction $<0.001$ ). When stratified by sex, the results in both males and females were also similar to those in the total population. Conclusion. Our data support a strongly positive combined effect of famine exposure and obesity parameters on hypertension in middle-aged and elderly Chinese.

\section{Introduction}

Hypertension or elevated blood pressure (BP) is a severe medical condition that significantly increases the risks of cardiovascular diseases (CVD) as well as other chronic diseases [1-3], such as congenital heart disease, heart failure, heart attack, peripheral vascular disease, stroke, and vascular disease. Hypertension modifiable risk factors [4-8] include age, sex, life stress, excessive drinking, high-salt diet, being overweight or obese, saturated fat and trans fats, tobacco use, low intake of fruits and vegetables, lack of physical activity, family history, low diet in vitamin D, advanced age, and coexisting diseases. Though the etiology of hypertension is complex, it was known as one of the strongest risk factors was overweight or obesity. Thus, increased body mass index (BMI) or centrally located body fat (especially waist circle (WC)) increases the risk of hypertension. In addition to known and probable risk factors for hypertension, early life malnutrition may also have an effect on hypertension.

It was hypothesized that early developmental adaptions in response to malnutrition in early life, which are the main factor for short-term survival, have adverse cardiovascular outcomes $[9,10]$. Historical famine exposure has provided a unique and natural opportunity to identify the hypothesis. Several previous studies [11-23] have provided evidence to support the relationship between famine exposure and increased risk of hypertension. Most studies [11, 13, 14, 16-23] have found that exposure to famine in early life could increase the risk of hypertension/BP in adulthood. Furthermore, exposure to famine has more deleterious effects on adult health for females than males $[12,15]$. However, other studies $[13,24,25]$ found no relationship between famine exposure and hypertension. Therefore, the relationship between famine exposure in early life and the risk of hypertension/BP needs to be further investigated. Moreover, studies also provided that malnutrition in early life [26-31] was more positively correlated with obesity among adults in late life. Generally speaking, it is not completed understood association and interaction analysis between famine exposure and obesity parameters (BMI and WC) and hypertension in the midage and older adult.

Given the limitations of previous studies, our study analyzed data from the China Health and Retirement Longitudinal Study Wave2011 (CHARLS Wave2011) and is aimed at exploring the individual and combined effects of famine exposure and obesity parameters on hypertension after adjustment for potential confounding variables.

\section{Methods}

2.1. Study Design and Setting. Data from the China Health and Retirement Longitudinal Study Wave2011 (CHARLS Wave2011) were used in our study. The CHARLS was a nationally representative longitudinal study conducted by the China Centre for Economic Research at Peking University [32]. In the CHARLS Wave2011, 13107 individuals were recruited for the baseline, after excluding participants with missing data, 12945 individuals were included in our study. All data are openly published as microdata at http://charls .pku.edu.cn/index/zh-cn.html with no direct contact with individuals. The Ethics Committee of the China Centre for Economic Research at Peking University approved the study; all individuals have provided informed consent before the data collection.

2.2. Individuals. The individuals of the study were selected from the CHARLS Wave2011 [32]. The mean age of CHARLS involved 12945 individuals was 59.33 years (standard deviation $(\mathrm{SD})=9.48$, ranged from 45 to 96 years). The mean age was 59.88 years $(\mathrm{SD}=9.36$, ranged from 45 to 90 years) in males and 58.83 years $(\mathrm{SD}=9.55$, ranged from 45 to 96 years) in females.

2.3. Baseline Characteristics. Baseline characteristics including age, sex $(0=$ male; $1=$ female $)$, marital status $(0=$ single; $1=$ married $)$, education ( $0=$ illiterate; $1=$ less than elementary school; $2=$ high school; $3=$ above vocational school), living place $(0=$ rural; $1=$ urban $)$, smoking status $(0=$ no; $1=$ former smoke; $2=$ current smoke), drinking status $(0=$ no; $1=$ less than once a month; $2=$ more than once a month), eating habit ( $0=\leq 2$ meals per day; $1=3$ meals per day; $2=\geq 4$ meals per day), social activities $(0=$ no; $1=$ yes $)$, experience of traumatic events $(0=$ no; $1=$ yes $)$, and physical exercise habit $(0=$ no; $1=$ less than regular physical exercises; 2 = regular physical exercises) were collected by self-report. Most variables were depending on our previous research studies [33-38]. 
2.4. Measurements. BMI was calculated based on the measured weight and height of the participants. Tapeline was localized at navel levels to read the WC at the end of exhalation. Using the standard China definition, BMI was categorized into three groups [39]: obesity $\left(B M I \geq 28 \mathrm{~kg} / \mathrm{m}^{2}\right)$, overweight $\left(24 \leq \mathrm{BMI}<28 \mathrm{~kg} / \mathrm{m}^{2}\right)$, and underweight and normal $\left(\right.$ BMI $\left.<24 \mathrm{~kg} / \mathrm{m}^{2}\right)$. Central obesity was defined as a WC [40] of $\geq 85 \mathrm{~cm}$ for females and $\geq 90 \mathrm{~cm}$ for males. Hypertension was defined as systolic blood pressure (SBP) of $\geq 140 \mathrm{mmHg}$ and/or diastolic blood pressure (DBP) of $\geq 90 \mathrm{mmHg}$; the definition has been used in our previous studies $[33,35,37,38]$.

2.5. Exposure Age and Exposed Groups. Famine exposure is set up on the previous Chinese famine study [41]; famine exposure was categorized into four exposure groups: noexposed group (birth year between 1963-01-01 and 196612-31), fetal-exposed group (birth year 1959-01-01 and 1962-12-31), childhood-exposed group (birth year 194901-01 and 1958-12-31), and adolescence/adult-exposed group (birth year between1921-01-01 and 1948-12-31).

2.6. Statistical Analysis. Analyses were conducted using SPSS software, version 22.0 (IBM SPSS, Armonk, NY, USA). The data are presented as mean \pm SD unless indicated otherwise. Means and SD (continuous data) were used to measure the continuous variable (age), and count and percentage were used to describe categorical variables (sex, education, marital status, living place, drinking status, smoking habit, eating habit, social activities, the experience of traumatic events, taking physical activity or exercise, famine exposure, BMI levels, WC levels, and hypertension categories). Betweengroup differences according to hypertension (hypertension and no-hypertension) were evaluated by the chi-square test (categorical data). Differences between baseline characteristics (sex, education, marital status, living place, drinking status, smoking habit, eating habit, social activities, the experience of traumatic events, and taking physical activity or exercise) and categories of famine exposure groups/BMI levels/WC levels were also evaluated using the chi-square test (categorical data). Age between groups was used by $t$ or F-test. Logistic regression models were used to compute ORs with accompanying 95\% CIs as estimates of associations of BMI/WC levels and exposure groups separately and in combination, with the prevalence of hypertension. Two-tailed $P<5 \%$ was considered to indicate statistical significance.

\section{Results}

Table 1 shows the basic characteristics of participants. A total of 12945 individuals were enrolled into the study; $6159(47.58 \%)$ participants and $6786(52.42 \%)$ participants were male and female, respectively. Among males, 676 (10.98\%) participants had been exposed to the Chinese famine during the fetal group, whereas 2448 (39.75\%) participants and $2233(36.26 \%)$ participants had been exposed to the famine during childhood and adolescence/adult groups, respectively. Among females, 872 (12.85\%) participants had been exposed to the Chinese famine during the fetal group, whereas $2653(39.10 \%)$ participants and 2129 (31.37\%) participants had been exposed to the famine during childhood and adolescence/adult groups, respectively. The distribution of living place and experience of traumatic events did not demonstrate significantly statistical differences among the four birth groups. On the other hand, the difference was observed in the distribution of age, sex, education, marital status, smoking status, drinking status, eating habit, social events, and physical exercise habit. Regarding the males, $4088(66.37 \%)$ were underweight and normal, 1572 (25.52\%) were overweight, and 499 (8.10\%) were obese, whereas 3646 (53.73\%), 2174 (32.04\%), and 966 (14.24\%) of the females were underweight and normal, overweight, and obese, respectively. Furthermore, significant differences in distribution were observed between BMI levels in all variables, including age, sex, education, marital status, living place, smoking status, drinking status, eating habit, social events, the experience of traumatic events, and physical exercise habit. Among the WC measures, 1839 (29.86\%) were central obesity in males and 3506 (51.67\%) in females. The proportions on the characteristics were statistically different between the WC groups except for age and marital status.

Table 2 shows the characteristics of study participants categorized by blood pressure status. Of the participants, $1920(31.17 \%)$ reported having hypertension in male and $2233(32.91 \%)$ in the female. Significant differences were observed in age, sex, education, marital status, living place, drinking status, the experience of traumatic events, famine groups, BMI levels, and WC groups $(P<0.05)$ between participants with and without hypertension.

Table 3 shows the separate associations of famine exposure, BMI, and central obesity with the prevalence of hypertension. Firstly, after controlling for confounding factors including age, education, marital status, living place, smoking status, drinking status, eating habit, social activities, the experience of traumatic events, taking physical activity or exercise, and famine exposure in a multivariable logistic regression model three, higher odds of prevalence of hypertension in the total population were observed with increasing levels of BMI (overweight vs. normal: 1.75 (95\% CI 1.60, 1.91); obesity vs. normal: 2.79 (95\% CI 2.48, 3.15)) and WC (overweight vs. normal: 1.42 (95\% CI 1.36, 1.48)) independently of famine groups only (BMI, $P$ for trend $<$ $0.001)$. When stratified by sex, the results of model three in both males and females were mostly similar to those in the total population. Secondly, after controlling for confounding factors including age, education, marital status, living place, smoking status, drinking status, eating habit, social activities, the experience of traumatic events, taking physical activity or exercise, and BMI in a multivariable logistic regression model three, higher odds of prevalence of hypertension in the total population were observed with famineexposed groups (fatal-exposed group vs. nonexposed group: 1.27 (95\% CI 1.08, 1.49); childhood-exposed group vs. nonexposed group: 1.64 (95\% CI 1.44, 1.87); adolescence/adultexposed group vs. nonexposed group: 3.06 (95\% CI 2.68, 3.50), $P$ for trend $<0.001)$ independently of BMI only $(P$ 


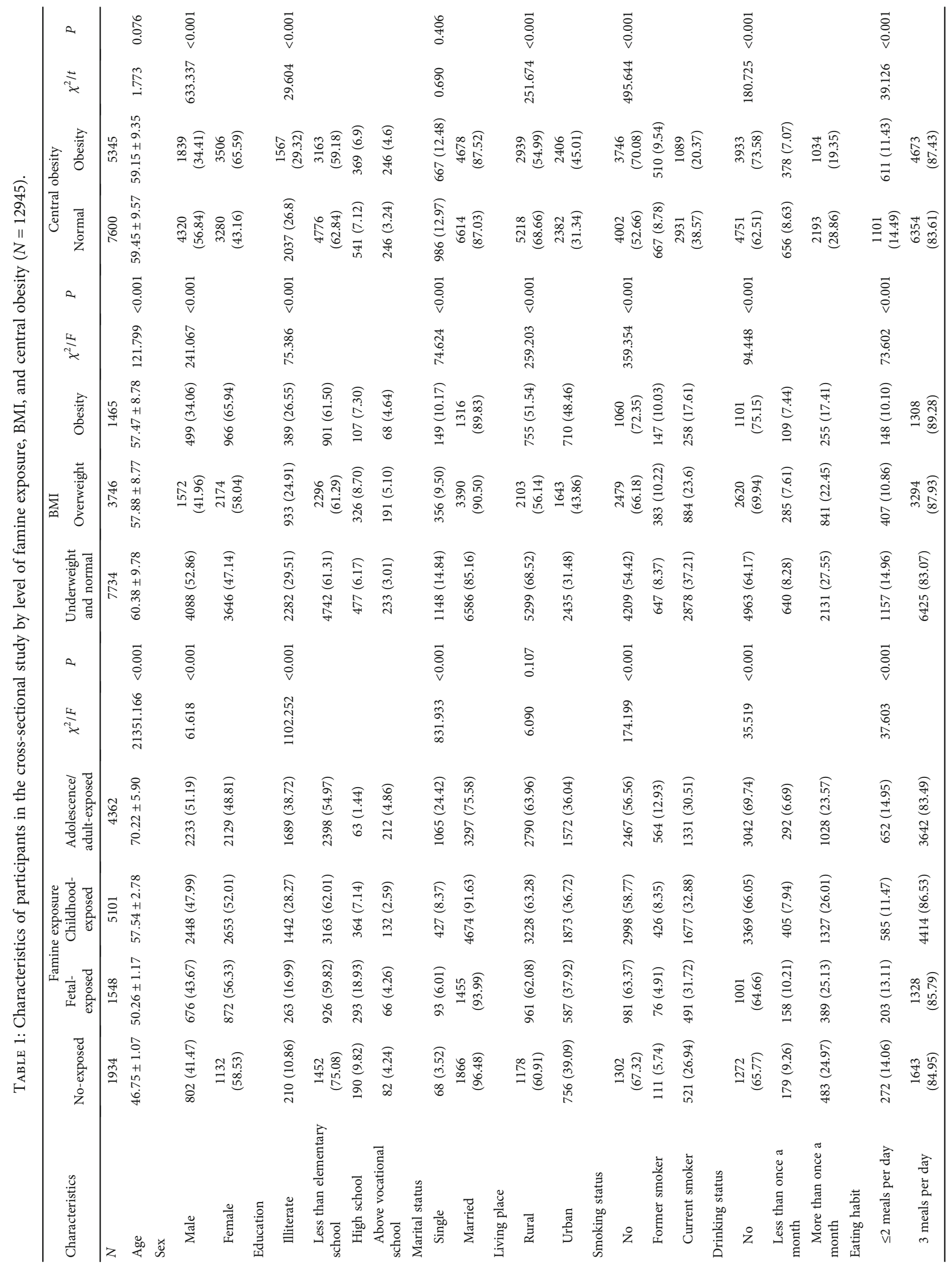




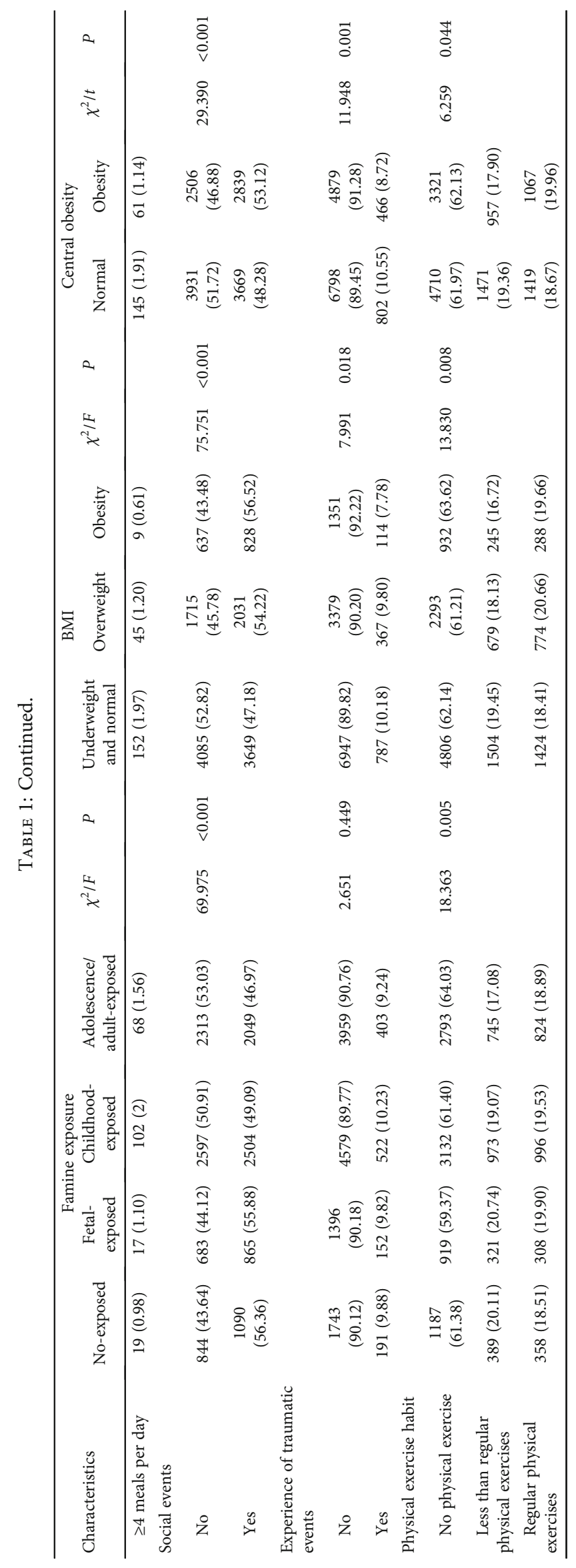


TABLE 2: Characteristics of study participants of cross-sectional study categorized by blood pressure status $(N=12945)$.

\begin{tabular}{|c|c|c|c|c|c|}
\hline Variables & $\begin{array}{l}\text { Without hypertension } \\
\qquad N=8792\end{array}$ & $\begin{array}{c}\text { Hypertension } \\
N=4153\end{array}$ & Total & $\chi^{2} / t$ & $P$ \\
\hline Age & $58.07 \pm 8.99$ & $62.00 \pm 9.92$ & $59.33 \pm 9.48$ & -21.720 & $<0.001$ \\
\hline Sex & & & & 4.445 & 0.035 \\
\hline Male & $4239(48.21)$ & $1920(46.23)$ & $6159(47.58)$ & & \\
\hline Female & $4553(51.79)$ & $2233(53.77)$ & $6786(52.42)$ & & \\
\hline \multicolumn{6}{|l|}{ Education } \\
\hline Illiterate & $2262(25.73)$ & $1342(32.31)$ & $3604(27.84)$ & 69.641 & $<0.001$ \\
\hline Less than elementary school & $5526(62.85)$ & $2413(58.1)$ & $7939(61.33)$ & & \\
\hline High school & $673(7.65)$ & $237(5.71)$ & $910(7.03)$ & & \\
\hline Above vocational school & $331(3.76)$ & $161(3.88)$ & $492(3.8)$ & & \\
\hline \multicolumn{6}{|l|}{ Marital status } \\
\hline Single & $919(10.45)$ & $734(17.67)$ & $1653(12.77)$ & & $<0.001$ \\
\hline Married & $7873(89.55)$ & $3419(82.33)$ & $11292(87.23)$ & 132.050 & \\
\hline \multicolumn{6}{|l|}{ Living place } \\
\hline Rural & $5671(64.5)$ & $2486(59.86)$ & $8157(63.01)$ & 26.072 & $<0.001$ \\
\hline Urban & $3121(35.5)$ & $1667(40.14)$ & $4788(36.99)$ & & \\
\hline \multicolumn{6}{|l|}{ Smoking status } \\
\hline No & $5265(59.88)$ & $2483(59.79)$ & $7748(59.85)$ & 4.187 & 0.123 \\
\hline Former smoke & $770(8.76)$ & $407(9.80)$ & $1177(9.09)$ & & \\
\hline Current smoke & $2757(31.36)$ & $1263(30.41)$ & $4020(31.05)$ & & \\
\hline \multicolumn{6}{|l|}{ Drinking status } \\
\hline No & $5852(66.56)$ & $2832(68.19)$ & $8684(67.08)$ & 11.233 & 0.004 \\
\hline Less than once a month & $750(8.53)$ & $284(6.84)$ & $1034(7.99)$ & & \\
\hline More than once a month & $2190(24.91)$ & $1037(24.97)$ & $3227(24.93)$ & & \\
\hline \multicolumn{6}{|l|}{ Eating habit } \\
\hline$\leq 2$ meals per day & $1144(13.01)$ & $568(13.68)$ & $1712(13.23)$ & 1.258 & 0.533 \\
\hline 3 meals per day & $7505(85.36)$ & $3522(84.81)$ & $11027(85.18)$ & & \\
\hline$\geq 4$ meals per day & $143(1.63)$ & $63(1.52)$ & $206(1.59)$ & & \\
\hline \multicolumn{6}{|l|}{ Social events } \\
\hline No & $4336(49.32)$ & $2101(50.59)$ & $6437(49.73)$ & 1.827 & 0.177 \\
\hline Yes & $4456(50.68)$ & $2052(49.41)$ & $6508(50.27)$ & & \\
\hline \multicolumn{6}{|l|}{ Experience of traumatic events } \\
\hline No & $7881(89.64)$ & $3796(91.40)$ & $11677(90.20)$ & 9.950 & 0.002 \\
\hline Yes & $911(10.36)$ & $357(8.60)$ & $1268(9.80)$ & & \\
\hline \multicolumn{6}{|l|}{ Physical exercise habit } \\
\hline No physical exercise & $5409(61.52)$ & $2622(63.14)$ & $8031(62.04)$ & 3.162 & 0.206 \\
\hline Less than regular physical exercises & $1675(19.05)$ & $753(18.13)$ & $2428(18.76)$ & & \\
\hline Regular physical exercises & $1708(19.43)$ & $778(18.73)$ & $2486(19.2)$ & & \\
\hline \multicolumn{6}{|l|}{ Famine exposure } \\
\hline No-exposed & $1536(17.47)$ & $398(9.58)$ & $1934(14.94)$ & 420.894 & $<0.001$ \\
\hline Fetal-exposed & $1167(13.27)$ & $381(9.17)$ & $1548(11.96)$ & & \\
\hline Childhood-exposed & $3609(41.05)$ & $1492(35.93)$ & $5101(39.41)$ & & \\
\hline Adolescence/adult-exposed & $2480(28.21)$ & $1882(45.32)$ & $4362(33.7)$ & & \\
\hline \multicolumn{6}{|l|}{ BMI } \\
\hline Underweight and normal & $5625(63.98)$ & $2109(50.78)$ & $7734(59.75)$ & 257.301 & $<0.001$ \\
\hline Overweight & $2387(27.15)$ & $1359(32.72)$ & $3746(28.94)$ & & \\
\hline Obesity & $780(8.87)$ & $685(16.49)$ & $1465(11.32)$ & & \\
\hline
\end{tabular}


TABLE 2: Continued.

\begin{tabular}{lccccc}
\hline Variables & $\begin{array}{c}\text { Without hypertension } \\
N=8792\end{array}$ & $\begin{array}{c}\text { Hypertension } \\
N=4153\end{array}$ & Total & $\chi^{2} / t$ & $P$ \\
\hline Central obesity & & & & & \\
$\quad$ Normal & $5647(64.23)$ & $1953(47.03)$ & $7600(58.71)$ & 344.333 & $<0.001$ \\
Obesity & $3145(35.77)$ & $2200(52.97)$ & $5345(41.29)$ & & \\
\hline
\end{tabular}

for trend $<0.001)$. When stratified by sex, the results of model three in both males and females were mostly similar to those in the total population. Lastly, after controlling for confounding factors including age, education, marital status, living place, smoking status, drinking status, eating habit, social activities, the experience of traumatic events, taking physical activity or exercise, and WC in a multivariable logistic regression model three, higher odds of prevalence of hypertension in the total population were observed with famine-exposed groups (fatal-exposed group vs. nonexposed group: 1.25 (95\% CI 1.06, 1.47); childhood-exposed group vs. nonexposed group: 1.52 (95\% CI 1.34, 1.73); adolescence/adult-exposed group vs. nonexposed group: 2.66 (95\% CI $2.33,3.03), P$ for trend $<0.001$ ) independently of WC only ( $P$ for trend $<0.001$ ). When stratified by sex, the results of model three in both males and females were mostly similar to those in the total population.

Table 4 shows the combined associations of obesity parameters and famine exposure with the prevalence of hypertension in males. Compared with the combination of the normal BMI/WC level and no-exposed famine group, all groups trended towards higher odds of prevalence of hypertension except the obesity; furthermore, in multivariable model one, the greatest increase in odds was observed for the adolescence/adult-exposed group and obesity combination (adolescence/adult-exposed group and obesity in BMI: OR 7.38; 95\% CI 4.81, 11.32; adolescence/adultexposed group and obesity in WC: OR 6.13; 95\% CI 4.54, 8.26). And similarly, in multivariable-adjusted model two, the highest odds of prevalence of hypertension were observed for the adolescence/adult exposed group and obesity combination (adolescence/adult-exposed group and obesity in BMI: OR 6.87; 95\% CI 4.47, 10.57; adolescence/adult-exposed group and obesity in WC: OR 5.75; 95\% CI $4.26,7.77)$. Additionally, in multivariable-adjusted model three, the highest odds of prevalence of hypertension were observed for the adolescence/adult-exposed group and obesity combination (adolescence/adult-exposed group and obesity in BMI: OR 7.30; 95\% CI 4.74, 11.25; adolescence/adult-exposed group and obesity in WC: OR 6.68 ; 95\% CI $4.92,9.07)$. Finally, combined associations of obesity parameters and famine exposure with the prevalence of hypertension were observed in males (Pinteraction $=<0.001$ ).

Table 5 shows the combined associations of obesity parameters and famine exposure with the prevalence of hypertension in females. Compared with the combination of the normal BMI/WC level and no-exposed famine group, all groups trended towards higher odds of prevalence of hypertension; furthermore, in multivariable model one, the greatest increase in odds was observed for the adolescence/a- dult-exposed group and obesity combination (adolescence/adult-exposed group and obesity in BMI: OR 10.38; $95 \%$ CI 7.26, 14.48; adolescence/adult-exposed group and obesity in WC: OR 7.59; 95\% CI 5.86, 9.84). And similarly, in multivariable-adjusted model two, the highest odds of prevalence of hypertension were observed for the adolescence/adult-exposed group and obesity combination (adolescence/adult-exposed group and obesity in BMI: OR 8.88; 95\% CI 6.18, 12.75; adolescence/adult-exposed group and obesity in WC: OR 6.58; 95\% CI 5.05, 8.58). Additionally, in multivariable-adjusted model three, the highest odds of prevalence of hypertension were observed for the adolescence/adult-exposed group and obesity combination (adolescence/adult-exposed group and obesity in BMI: OR 8.89 ; 95\% CI 6.19, 12.78; adolescence/adult-exposed group and obesity in WC: OR 6.59; 95\% CI 5.05, 8.59). Finally, combined associations of obesity parameters and famine exposure with the prevalence of hypertension were observed in females $(P$ interaction $<0.001)$.

Table 6 shows the combined associations of obesity parameters and famine exposure with the prevalence of hypertension in the total population. Compared with the combination of the normal BMI/WC level and no-exposed famine group, all groups trended towards higher odds of prevalence of hypertension; furthermore, in multivariable model one, the greatest increase in odds was observed for the adolescence/adult-exposed group and obesity combination (adolescence/adult-exposed group and obesity in BMI: OR 9.04; 95\% CI 6.89, 11.86; adolescence/adult-exposed group and obesity in WC: OR 7.05; 95\% CI 5.80, 8.56). And similarly, in multivariable-adjusted model two, the highest odds of prevalence of hypertension were observed for the adolescence/adult-exposed group and obesity combination (adolescence/adult-exposed group and obesity in BMI: OR 7.94; 95\% CI 6.03, 10.44; adolescence/adultexposed group and obesity in WC: OR 6.29; 95\% CI 5.16, 7.66). Additionally, in multivariable-adjusted model three, the highest odds of prevalence of hypertension were observed for the adolescence/adult-exposed group and obesity combination (adolescence/adult-exposed group and obesity in BMI: OR 8.13; 95\% CI 6.18, 10.71; adolescence/adultexposed group and obesity in WC: OR 6.36; $95 \%$ CI 5.22 , 7.75). Finally, combined associations of obesity parameters and famine exposure with the prevalence of hypertension were observed in the total population (Pinteraction $<0.001$ ).

\section{Discussion}

In the study, we found that the individuals who had been exposed to famine in early life had an increased risk of 


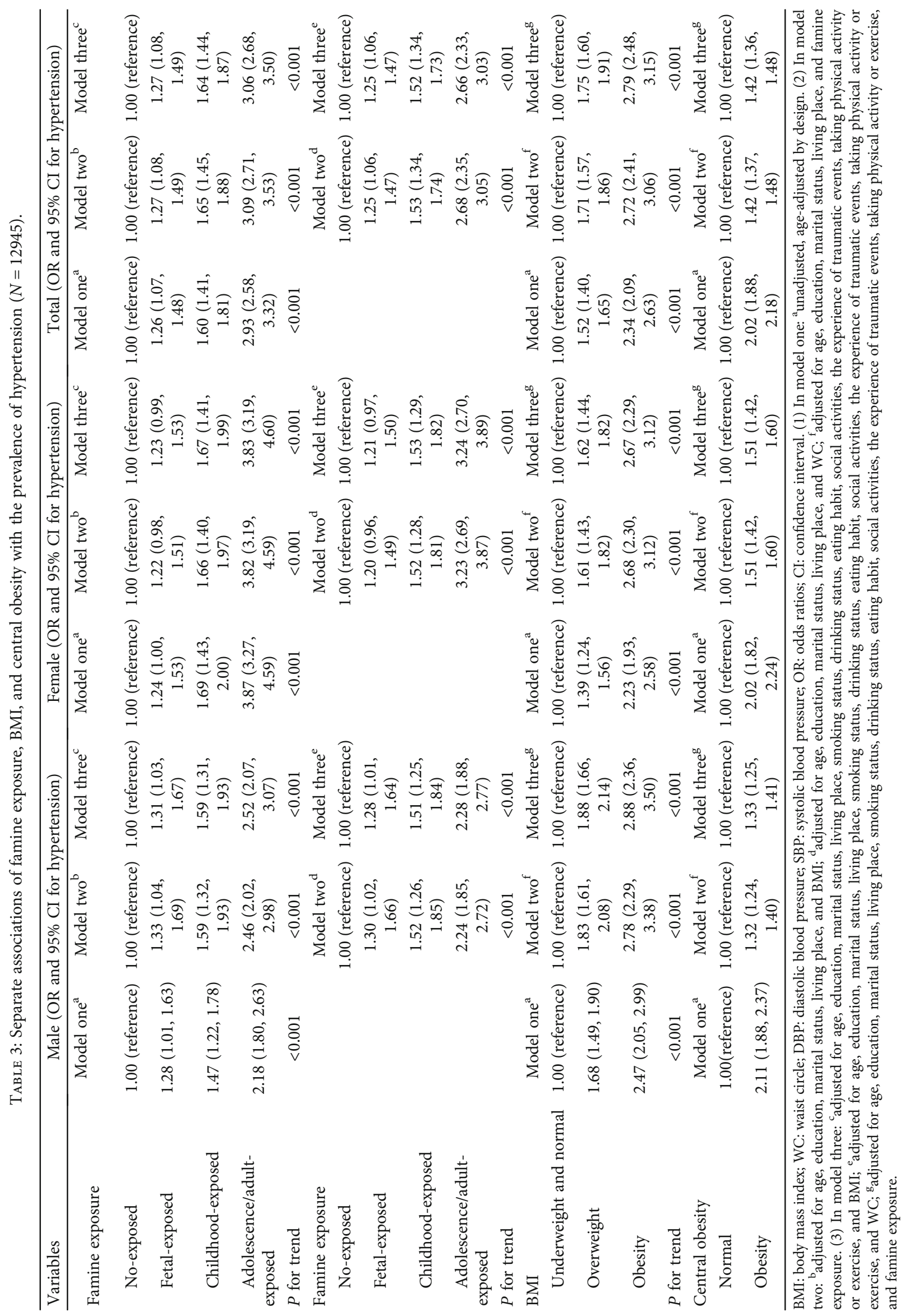




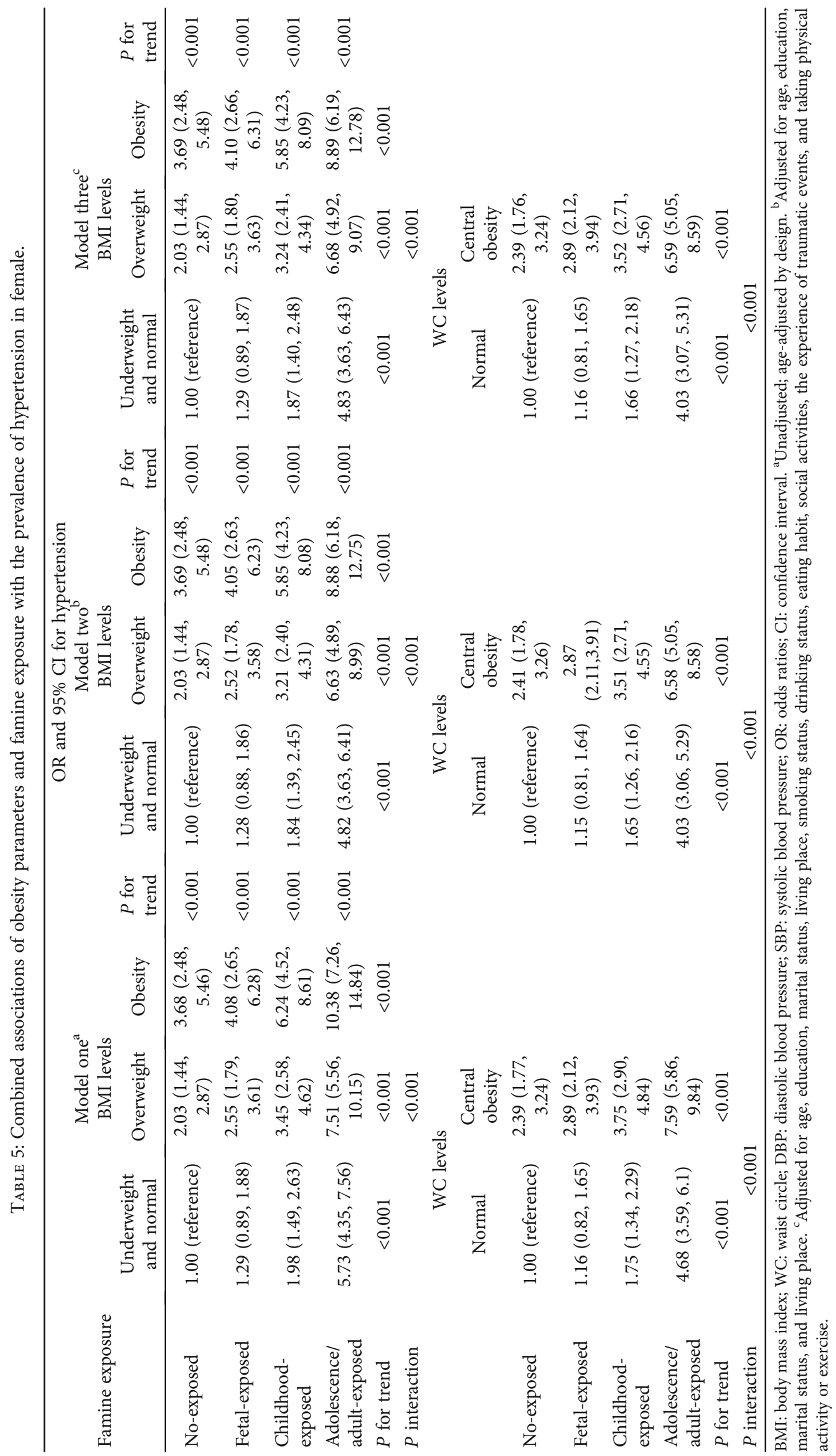




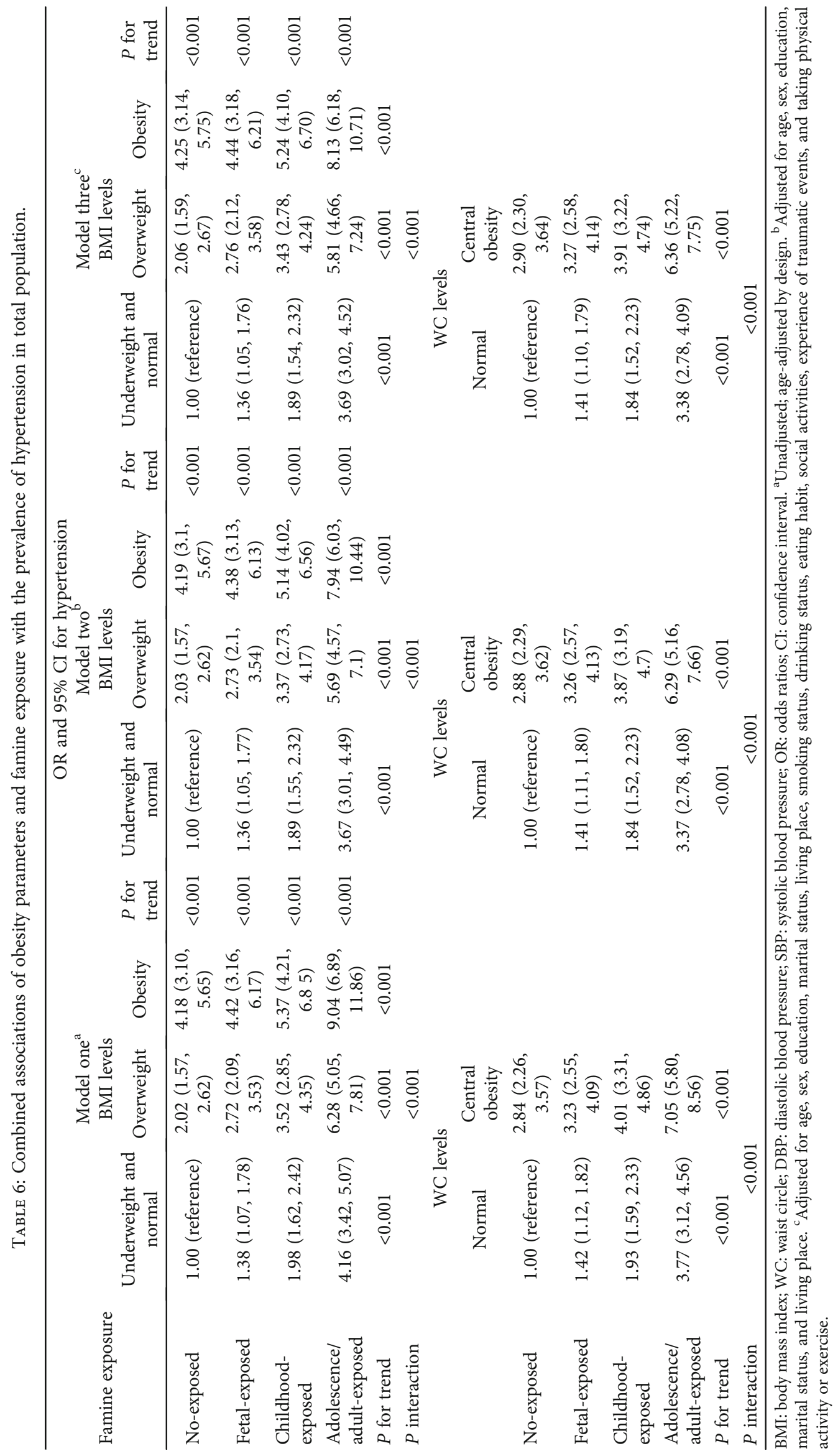


hypertension in adults. After adjustment for the full set of potential confounders, including age, education, marital status, living place, smoking status, drinking status, eating habit, social activities, the experience of traumatic events, taking physical activity or exercise, and obesity parameters (BMI or WC), the associations still can be found in males and females. Additionally, our study found that there were linear trends in the associations of BMI with hypertension. After adjustment for observed potential confounders, the associations still existed both in males and females. In summary, our study supports a strongly positive combined effect of famine exposure and obesity parameters on hypertension in middle-aged and elderly Chinese. When stratified by sex, similar results were found with respect to the association.

The Chinese famine of 1959-61 caused over 30 million excess deaths [42]. A large number of such studies have explored the associations of famine exposure during early life with the risk of hypertension in adults, and there were no consistent associations observed for these studies. Therefore, our study attempted to explore the associations between obesity parameters (BMI or WC) and hypertension based on a national study from CHARLS2011. In conclusion, the findings from our study support a strongly positive combined effect of famine exposure and obesity parameters (BMI or WC) on hypertension in middle-aged and elderly Chinese. Both nutrition intervention for exposure to the famine in early life and weight control in later life may be required to substantially reduce the risk of hypertension in later life.

The effect of the worst famine to hypertension may be masked, however, by a selection effect of survivors who might be healthier than the frail members more likely to survive. The finding is in line with Darwin's theory of survival of the fittest [43]. Individuals who were exposed to famine in early life should decrease the risk of hypertension in adults. However, this was not observed in our research. The reason for the inconsistency may be due to the environmental changes. When facing the later "rich" environment, the risk of hypertension may be increased.

Our results are partly in line with several previous studies. Although the Dutch famine and the Leningrad siege study [44-46] have generally agreed that early-life exposure to famine was not associated with hypertension, most current published research findings $[11,12,14-23]$ in China indicated that exposure to famine in early life increased the risk of hypertension. However, it was found there was no association between the Chinese famine and hypertension risk in Chongqing [24]. Such discrepancies between these studies may be a result of methodological differences in definitions of the different sample selection effect and famine exposure groups. Additionally, these studies have been criticized for not being adjusted to the confounding bias of age. To control the age confounding, we categorized the famine exposure into four exposure groups based on the birth year and we also combined the no-exposure as the reference group to identify the effect of the fetal-exposed group, childhood-exposed group, and adolescence/adult-exposed group. Our study found that early famine exposure was associated with an increased risk of hypertension. The sex difference of early life famine exposure and hypertension were common in other studies $[15,17]$. Furthermore, exposure to famine during early life exerted more deleterious effects on females than males. This could be explained by the fact the female may suffer more than males during early life, because of the dominance of a patriarchal mentality in China [47]. The potential mechanisms of the associations between famine exposure in early life and the increased risk of hypertension in later life were still not fully understood. Animal experiments $[48,49]$ have proved that malnutrition in early life could result in elevated BP in later life. Additionally, epigenetic might play a role in the association between famine exposure in early life and hypertension in late life $[50,51]$.

In our research, participants who were overweight/obese and exposed to famine in adolescence/adult tended to have a higher risk of hypertension. The results indicated the good nutrition in adults did not match poor nutrition in early life, which might elevate the relative risk of hypertension in later life [52]. Furthermore, our data support a strongly positive combined effect of famine exposure and obesity parameters on hypertension in middle-aged and elderly Chinese. However, the previous studies focused on the relationship between famine exposure and health outcomes in late adolescence and adulthood. Most of the previous studies meant exactly that famine exposure was at a higher risk for health outcomes in late adolescence and adulthood. Exposure to Chinese famine in early life was related to increased risk of metabolic syndrome [41, 44, 53-57], weight gain [26-31], diabetes [58-73], hypertension [11, 12, 14-23], cognitive decline [74-80], and depressive syndrome [42, 76, 81, 82]. In addition, our study found that there were linear trends in the associations of BMI with hypertension which was consistent with our previous study [83]. However, several studies proved that there were different associations of body mass index with health outcomes, such as U- [84-88], J[89-95], and reverse J-shape [96, 97]. A U- or a J-shaped association between BMI and cardiovascular events is often described. This U-shaped association may result from the effect of medication use or unintentional weight loss. By contrast, patients with other severe heart diseases or undergoing cardiac surgery presented a reverse J-shape suggesting the low body mass index associated with the highest mortality [98].

Though so many studies have explored the association analysis between famine exposure/obesity parameters and $\mathrm{BP}$, there were only two studies that explored the combined effect of obesity parameters on the relation between famine exposure and hypertension. Yu et al. [19] found that interactions between famine and obesity on hypertension prevalence risk were not observed. In contrast, Li et al. [11] reported that a stronger interaction between obesity and famine exposure with regard to BP among individuals who were exposed to famine during fetal life and had a western dietary pattern in adults was observed. Interestingly, our data support a strongly positive combined effect of famine exposure and obesity parameters on hypertension in middle-aged and elderly Chinese. The difference between our research and others may be due to the different populations, different definitions of famine exposure cohort, and 
different confounding variables by controlling. The individuals in our study were midaged and elderly Chinese, where the mean age at recruitment was older than in $\mathrm{Yu}$ et al.' $\mathrm{s}$ study, and the level of socioeconomic development also made some contribution to ontogenetic development. In addition, the participants were similar in China and its socioeconomic background, and this phenomenon could be explained by the cumulative effect.

Several limitations have to be taken into account as well. Firstly, selection bias was to be considered: famine may weed out the frail members and leave the healthier ones. Secondly, individual famine exposure data have not been collected. Lastly, not all families were equally affected by famine exposure. However, our study provided a large data that could be explored further in the combined effect of famine exposure and obesity parameters (BMI or WC) on hypertension. Moreover, a significant strength of the study is the large sample of 12945 middle-aged and older Chinese. Another strength is the analytical method of controlling for a number of confounders.

\section{Conclusions}

Our data support a strongly positive combined effect of famine exposure and obesity parameters on hypertension in middle-aged and elderly Chinese. Both nutrition intervention for exposure to the famine in early life and weight reduction in later life may be required to substantially reduce the risk of hypertension in later life.

\section{Abbreviations}

WHO: World Health Organization

CHARLS: China Health and Retirement Longitudinal Study

BMI: $\quad$ Body mass index

WC: Waist circle

BP: $\quad$ Blood pressure

DBP: Diastolic blood pressure

SBP: $\quad$ Systolic blood pressure

M: $\quad$ Mean

B: $\quad$ Unstandardized

CDC: Centers for Disease Control and Prevention

NSFC: The National Natural Science Foundation of China

NIA: National Institute on Aging

WB: $\quad$ World Bank

UA: Urinalysis.

\section{Data Availability}

All data are openly published as microdata at http://charls .pku.edu.cn/index/zh-cn.html with no direct contact with all participants.

\section{Consent}

Completion of all author declaration and consent to publish form is required.

\section{Disclosure}

The authors declare that they have no potential conflict of interest relevant to the study.

\section{Conflicts of Interest}

The authors declare that they have no conflicts of interest.

\section{Authors' Contributions}

Lin Zhang conceived and designed the research, wrote the paper, and analyzed the data. Lin Zhang, Liu Yang, Congzhi Wang, Ting Yuan, Dongmei Zhang, Huanhuan Wei, Jing Li, Yunxiao Lei, Lu Sun, Xiaoping Li, Ying Hua, Hengying Che, and Yuanzhen Li revised the paper. Yuanzhen Li and Hengying Che contributed equally to this work.

\section{Acknowledgments}

This work was supported by the NSFC (70910107022 and 71130002) and National Institute on Aging (R03TW008358-01 and R01-AG037031-03S1), World Bank (7159234), and the Support Program for Outstanding Young Talents from the Universities and Colleges of Anhui Province for Lin Zhang and the Key Research Base of Humanities and Social Sciences of Universities of Anhui Province (SK2019A0223) for Wang Congzhi. The authors would like to thank the members of the CHARLS as well as all participants for their contribution.

\section{References}

[1] I. de Kouchkovsky, J. Mayfield, and J. Kohlwes, "Hypertension in young adults and subsequent cardiovascular disease," JAMA, vol. 321, no. 13, p. 1310, 2019.

[2] J. Gutierrez, A. Alloubani, M. Mari, and M. Alzaatreh, "Cardiovascular disease risk factors: hypertension, diabetes mellitus and obesity among Tabuk citizens in Saudi Arabia," Open Cardiovascular Medicine Journal, vol. 12, no. 1, pp. 41-49, 2018.

[3] J. E. James, "Hypertension control and cardiovascular disease," Lancet, vol. 389, no. 10065, p. 154, 2017.

[4] S. Abdulsalam, A. Olugbenga-Bello, O. Olarewaju, and I. Abdus-Salam, "Sociodemographic correlates of modifiable risk factors for hypertension in a rural local government area of Oyo state south west Nigeria," International Journal of Hypertension, vol. 2014, Article ID 842028, 9 pages, 2014.

[5] L. Ke, J. Ho, J. Feng et al., "Modifiable risk factors including sunlight exposure and fish consumption are associated with risk of hypertension in a large representative population from Macau," The Journal of Steroid Biochemistry and Molecular Biology, vol. 144, pp. 152-155, 2014.

[6] R. Ibekwe, "Modifiable risk factors of hypertension and sociodemographic profile in Oghara, delta state; prevalence and correlates," Annals of Medical and Health Sciences Research, vol. 5, no. 1, pp. 71-77, 2015.

[7] Z. Pilakkadavath and M. Shaffi, "Modifiable risk factors of hypertension: a hospital-based case-control study from Kerala, India," Journal of Family Medicine and Primary Care, vol. 5, no. 1, pp. 114-119, 2016. 
[8] H. M. Mamudu, T. K. Paul, L. Wang et al., "Association between multiple modifiable risk factors of cardiovascular disease and hypertension among asymptomatic patients in central Appalachia," Southern Medical Journal, vol. 110, no. 2, pp. 90-96, 2017.

[9] P. D. Gluckman, M. A. Hanson, C. Cooper, and K. L. Thornburg, "Effect of in utero and early-life conditions on adult health and disease," The New England Journal of Medicine, vol. 359, no. 1, pp. 61-73, 2008.

[10] B. T. Alexander, "Fetal programming of hypertension," American Journal of Physiology-Regulatory, Integrative and Comparative Physiology, vol. 290, no. 1, pp. R1-R10, 2006.

[11] Y. Li, V. W. Jaddoe, L. Qi et al., "Exposure to the Chinese famine in early life and the risk of hypertension in adulthood," Journal of Hypertension, vol. 29, no. 6, pp. 1085-1092, 2011.

[12] A. F. M. van Abeelen, S. R. de Rooij, C. Osmond et al., "The sex-specific effects of famine on the association between placental size and later hypertension," Placenta, vol. 32, no. 9, pp. 694-698, 2011.

[13] D. Carroll, A. T. Ginty, R. C. Painter, T. J. Roseboom, A. C. Phillips, and S. R. de Rooij, "Systolic blood pressure reactions to acute stress are associated with future hypertension status in the Dutch Famine Birth Cohort Study," International Journal of Psychophysiology: official journal of the International Organization of Psychophysiology, vol. 85, no. 2, pp. 270-273, 2012.

[14] P. X. Wang, J. J. Wang, Y. X. Lei, L. Xiao, and Z. C. Luo, "Impact of fetal and infant exposure to the Chinese Great Famine on the risk of hypertension in adulthood," PLoS One, vol. 7, no. 11, article e49720, 2012.

[15] H. Chen, W. N. Nembhard, and H. G. Stockwell, "Sex-specific effects of fetal exposure to the 1959-1961 Chinese famine on risk of adult hypertension," Maternal and Child Health Journal, vol. 18, no. 3, pp. 527-533, 2014.

[16] Z. Wang, C. Li, Z. Yang, Z. Zou, and J. Ma, "Infant exposure to Chinese famine increased the risk of hypertension in adulthood: results from the China Health and Retirement Longitudinal Study," BMC Public Health, vol. 16, no. 1, p. 435, 2016.

[17] L. Liu, X. Xu, H. Zeng et al., "Increase in the prevalence of hypertension among adults exposed to the Great Chinese Famine during early life," Environmental Health and Preventive Medicine, vol. 22, no. 1, p. 64, 2017.

[18] L. Wu, X. Feng, A. He, Y. Ding, X. Zhou, and Z. Xu, "Prenatal exposure to the Great Chinese Famine and mid-age hypertension," PLoS One, vol. 12, no. 5, article e0176413, 2017.

[19] C. Yu, J. Wang, Y. Li et al., "Exposure to the Chinese famine in early life and hypertension prevalence risk in adults," PLoS One, vol. 35, pp. 63-68, 2017.

[20] L. Liu, X. Xu, H. Zeng et al., "Correction to: increase in the prevalence of hypertension among adults exposed to the great Chinese famine during early life," Environmental Health and Preventive Medicine, vol. 23, no. 1, p. 11, 2018.

[21] Z. Shi, S. J. Nicholls, A. W. Taylor, D. J. Magliano, S. Appleton, and P. Zimmet, "Early life exposure to Chinese famine modifies the association between hypertension and cardiovascular disease," Journal of Hypertension, vol. 36, no. 1, pp. 54-60, 2018.

[22] X. Xin, J. Yao, F. Yang, and D. Zhang, "Famine exposure during early life and risk of hypertension in adulthood: a metaanalysis," Critical Reviews in Food Science and Nutrition, vol. 58, no. 14, pp. 2306-2313, 2018.
[23] R. Zhao, X. Duan, Y. Wu, Q. Zhang, and Y. Chen, “Association of exposure to Chinese famine in early life with the incidence of hypertension in adulthood: a 22 -year cohort study," Nutrition, Metabolism, and Cardiovascular Diseases: NMCD, vol. 29, no. 11, pp. 1237-1244, 2019.

[24] J. P. Chen, B. Peng, L. Tang et al., "Fetal and infant exposure to the Chinese famine increases the risk of fatty liver disease in Chongqing, China," Journal of Gastroenterology and Hepatology, vol. 31, no. 1, pp. 200-205, 2016.

[25] J. Zhou, J. Sheng, Y. Fan et al., "The effect of Chinese famine exposure in early life on dietary patterns and chronic diseases of adults," Public Health Nutrition, vol. 22, no. 4, pp. 603-613, 2019.

[26] L. Liu, Z. C. Pang, J. P. Sun et al., "Exposure to famine in early life and the risk of obesity in adulthood in Qingdao: evidence from the 1959-1961 Chinese famine," Nutrition, Metabolism, and Cardiovascular Diseases: NMCD, vol. 27, no. 2, pp. 154160, 2017.

[27] X. Chang, P. Song, M. Wang, and L. An, "The risks of overweight, obesity and abdominal obesity in middle age after exposure to famine in early life: evidence from the China's 1959-1961 famine," The Journal of Nutrition, Health \& Aging, vol. 22, no. 10, pp. 1198-1204, 2018.

[28] R. Meng, J. Lv, C. Yu et al., "Prenatal famine exposure, adulthood obesity patterns and risk of type 2 diabetes," International Journal of Epidemiology, vol. 47, no. 2, pp. 399-408, 2018.

[29] J. Zhou, L. Zhang, P. Xuan et al., "The relationship between famine exposure during early life and body mass index in adulthood: a systematic review and meta-analysis," PLoS One, vol. 13, no. 2, article e0192212, 2018.

[30] Z. Fang, C. Chen, H. Wang, and K. Tang, "Association between fetal exposure to famine and anthropometric measures in adulthood: a regression discontinuity approach," Obesity, vol. 28, no. 5, pp. 962-969, 2020.

[31] C. Song, M. Wang, Z. Chen et al., "Fetal exposure to Chinese famine increases obesity risk in adulthood," International Journal of Environmental Research and Public Health, vol. 17, no. 10, p. 3649, 2020.

[32] C. Li, T. Liu, W. Sun, L. Wu, and Z. Y. Zou, "Prevalence and risk factors of arthritis in a middle-aged and older Chinese population: the China health and retirement longitudinal study," Rheumatology, vol. 54, no. 4, pp. 697-706, 2015.

[33] L. Zhang, K. Liu, H. Li et al., "Relationship between body mass index and depressive symptoms: the "fat and jolly" hypothesis for the middle-aged and elderly in China," BMC Public Health, vol. 16, no. 1, p. 1201, 2016.

[34] L. Zhang, J. L. Li, L. L. Zhang, L. L. Guo, H. Li, and D. Li, "No association between C-reactive protein and depressive symptoms among the middle-aged and elderly in China Evidence from the China Health and Retirement Longitudinal Study," Medicine, vol. 97, 2018.

[35] L. Zhang, L. Yang, C. Wang et al., "Individual and combined association analysis of famine exposure and serum uric acid with hypertension in the mid-aged and older adult: a population-based cross-sectional study," BMC Cardiovascular Disorders, vol. 21, no. 1, 2021.

[36] L. Zhang, J. L. Li, L. L. Zhang et al., "Relationship between adiposity parameters and cognition: the "fat and jolly" hypothesis in middle-aged and elderly people in China," Medicine, vol. 98, no. 10, p. e14747, 2019. 
[37] L. Zhang, J. L. Li, L. L. Zhang, L. L. Guo, H. Li, and D. Li, "Body mass index and serum uric acid level," Medicine (Baltimore), vol. 99, no. 9, article e19418, 2020.

[38] L. Zhang, J. L. Li, L. L. Guo, H. Li, D. Li, and G. Xu, "The interaction between serum uric acid and triglycerides level on blood pressure in middle-aged and elderly individuals in China: result from a large national cohort study," BMC Cardiovascular Disorders, vol. 20, no. 1, p. 174, 2020.

[39] B. F. Zhou, "Effect of body mass index on all-cause mortality and incidence of cardiovascular diseases-report for metaanalysis of prospective studies open optimal cut-off points of body mass index in Chinese adults," Biomedical and Environmental Sciences: BES, vol. 15, pp. 245-252, 2002.

[40] H. C. Zhou, Y. X. Lai, Z. Y. Shan et al., "Effectiveness of different waist circumference cut-off values in predicting metabolic syndrome prevalence and risk factors in adults in China," Biomedical and Environmental Sciences: BES, vol. 27, pp. 325-334, 2014.

[41] N. Wang, X. Wang, Q. Li et al., "The famine exposure in early life and metabolic syndrome in adulthood," Clinical Nutrition (Edinburgh, Scotland), vol. 36, no. 1, pp. 253-259, 2017.

[42] Y. Li, L. Zhao, D. Yu, and G. Ding, "Exposure to the Chinese famine in early life and depression in adulthood," Psychology, Health \& Medicine, vol. 23, no. 8, pp. 952-957, 2018.

[43] S. Song, "Does famine have a long-term effect on cohort mortality? Evidence from the 1959-1961 great leap forward famine in China," Journal of Biosocial Science, vol. 41, no. 4, pp. 469491, 2009.

[44] S. R. de Rooij, R. C. Painter, F. Holleman, P. M. Bossuyt, and T. J. Roseboom, "The metabolic syndrome in adults prenatally exposed to the Dutch famine," The American Journal of Clinical Nutrition, vol. 86, no. 4, pp. 1219-1224, 2007.

[45] A. D. Stein, P. A. Zybert, K. van der Pal-de Bruin, and L. H. Lumey, "Exposure to famine during gestation, size at birth, and blood pressure at age $59 \mathrm{y}$ : evidence from the Dutch famine," European Journal of Epidemiology, vol. 21, no. 10, pp. 759-765, 2006.

[46] S. A. Stanner, K. Bulmer, C. Andrès et al., "Does malnutrition in utero determine diabetes and coronary heart disease in adulthood? Results from the Leningrad siege study, a cross sectional study," BMJ, vol. 315, no. 7119, pp. 1342-1348, 1997.

[47] P. Khanal, L. Johnsen, A. M. Axel et al., "Long-term impacts of foetal malnutrition followed by early postnatal obesity on fat distribution pattern and metabolic adaptability in adult sheep," PLoS One, vol. 11, no. 6, article e0156700, 2016.

[48] V. Bol, F. Desjardins, B. Reusens, J. L. Balligand, and C. Remacle, "Does early mismatched nutrition predispose to hypertension and atherosclerosis, in male mice?," PloS One, vol. 5, no. 9, article e12656, 2010.

[49] S. Y. Bai, D. I. Briggs, and M. H. Vickers, "Increased systolic blood pressure in rat offspring following a maternal lowprotein diet is normalized by maternal dietary choline supplementation," Journal of Developmental Origins of Health and Disease, vol. 3, no. 5, pp. 342-349, 2012.

[50] A. Kubaszek, A. Markkanen, J. G. Eriksson et al., "The association of the K121Q polymorphism of the plasma cell glycoprotein-1 gene with type 2 diabetes and hypertension depends on size at birth," The Journal of Clinical Endocrinology and Metabolism, vol. 89, no. 5, pp. 2044-2047, 2004.

[51] K. M. Aagaard-Tillery, K. Grove, J. Bishop et al., "Developmental origins of disease and determinants of chromatin structure: maternal diet modifies the primate fetal epigenome," Journal of Molecular Endocrinology, vol. 41, pp. 91-102, 2008.

[52] A. Singhal and A. Lucas, "Early origins of cardiovascular disease: is there a unifying hypothesis?," Lancet, vol. 363, no. 9421, pp. 1642-1645, 2004.

[53] Y. Peng, M. Hai, P. Li, and Y. Chen, "Association of exposure to Chinese famine in early life with the risk of metabolic syndrome in adulthood," Annals of Nutrition \& Metabolism, vol. 76, no. 2, pp. 140-146, 2020.

[54] X. L. Feng, J. H. Liu, S. Yan et al., "Prenatal exposure to the Chinese famine and the risk of metabolic syndrome in adulthood across consecutive generations," Journal of Diabetes Research, vol. 74, 1236 pages, 2020.

[55] F. Ning, J. Ren, X. Song et al., "Famine exposure in early life and risk of metabolic syndrome in adulthood: comparisons of different metabolic syndrome definitions," vol. 2019, Article ID 7954856, pp. 1-9, 2019.

[56] X. Zheng, Y. Wang, W. Ren et al., "Risk of metabolic syndrome in adults exposed to the great Chinese famine during the fetal life and early childhood," European Journal of Clinical Nutrition, vol. 66, no. 2, pp. 231-236, 2012.

[57] Y. Li, V. W. Jaddoe, L. Qi et al., "Exposure to the chinese famine in early life and the risk of metabolic syndrome in adulthood," Diabetes Care, vol. 34, no. 4, pp. 1014-1018, 2011.

[58] R. C. W. Ma, P. Zimmet, H. Liu et al., "Association of famine exposure with the risk of type 2 diabetes: a meta-analysis," Journal of Diabetes, vol. 39, pp. 1717-1723, 2020.

[59] J. Lu, "Early life famine exposure, ideal cardiovascular health metrics, and risk of incident diabetes: findings from the $4 \mathrm{C}$ study," The British Journal of Nutrition, vol. 43, pp. 19021909, 2020.

[60] L. Ji and Z. Shi, "Early life exposure to 1959-1961 Chinese famine exacerbates association between diabetes and cardiovascular disease," Nature Reviews. Endocrinology, vol. 12, pp. 134$141,2020$.

[61] X. Hu, J. Wen, W. Yu et al., “Associations of early-life exposure to famine with abdominal fat accumulation are independent of family history of diabetes and physical activity," British Journal of Nutrition, vol. 125, no. 8, pp. 943-950, 2021.

[62] C. Li, E. W. Tobi, B. T. Heijmans, and L. H. Lumey, "The effect of the Chinese famine on type 2 diabetes mellitus epidemics," Nature Reviews Endocrinology, vol. 15, no. 6, pp. 313-314, 2019.

[63] C. Li and L. H. Lumey, "Interaction or mediation by adult obesity of the relation between fetal famine exposure and type 2 diabetes?," Nature Reviews Endocrinology, vol. 48, pp. 654656, 2019.

[64] Z. Wang, Z. Zou, Z. Yang et al., "The association between fetalstage exposure to the China famine and risk of diabetes mellitus in adulthood: results from the China health and retirement longitudinal study," BMC Public Health, vol. 18, no. 1, p. 1205, 2018.

[65] Y. Sun, L. Zhang, W. Duan, X. Meng, and C. Jia, "Association between famine exposure in early life and type 2 diabetes mellitus and hyperglycemia in adulthood: results from the China Health And Retirement Longitudinal Study(CHARLS)," Journal of Diabetes, vol. 10, no. 9, pp. 724-733, 2018.

[66] T. Li, Y. Zhang, J. Wang et al., “All-cause mortality risk associated with long-term exposure to ambient $\mathrm{PM}_{2 * 5}$ in China: a cohort study," The Lancet Public health, vol. 3, no. 10, pp. e470-e477, 2018. 
[67] N. Wang, J. Cheng, B. Han et al., "Exposure to severe famine in the prenatal or postnatal period and the development of diabetes in adulthood: an observational study," Diabetologia, vol. 60, no. 2, pp. 262-269, 2017.

[68] J. Li, S. Liu, S. Li et al., "Prenatal exposure to famine and the development of hyperglycemia and type 2 diabetes in adulthood across consecutive generations: a populationbased cohort study of families in Suihua, China," The American Journal of Clinical Nutrition, vol. 105, no. 1, pp. 221227, 2017.

[69] J. Wang, Y. Li, X. Han et al., "Exposure to the Chinese famine in childhood increases type 2 diabetes risk in adults," The Journal of Nutrition, vol. 146, no. 11, pp. 2289-2295, 2016.

[70] L. H. Lumey, M. D. Khalangot, and A. M. Vaiserman, “Association between type 2 diabetes and prenatal exposure to the Ukraine famine of 1932-33: a retrospective cohort study," The Lancet. Diabetes \& Endocrinology, vol. 3, no. 10, pp. 787-794, 2015.

[71] A. F. van Abeelen, S. G. Elias, P. M. Bossuyt et al., "Famine exposure in the young and the risk of type 2 diabetes in adulthood," Diabetes, vol. 61, no. 9, pp. 2255-2260, 2012.

[72] F. Portrait, E. Teeuwiszen, and D. Deeg, "Early life undernutrition and chronic diseases at older ages: the effects of the Dutch famine on cardiovascular diseases and diabetes," Social Science \& Medicine, vol. 73, no. 2011, pp. 711-718, 1982.

[73] Y. Li, Y. He, L. Qi et al., "Exposure to the Chinese famine in early life and the risk of hyperglycemia and type 2 diabetes in adulthood," Diabetes, vol. 59, no. 10, pp. 2400-2406, 2010.

[74] G. Arage, T. Belachew, M. Abera, F. Abdulhay, M. Abdulahi, and K. Hassen Abate, "Consequences of early life exposure to the 1983-1985 Ethiopian great famine on cognitive function in adults: a historical cohort study," BMJ Open, vol. 10, no. 9, article e038977, 2020.

[75] H. Rong, X. Lai, E. Mahmoudi, and H. Fang, "Early-life exposure to the Chinese famine and risk of cognitive decline," Journal of Clinical Medicine, vol. 8, no. 4, p. 484, 2019.

[76] C. Li, T. Miles, L. Shen et al., "Early-life exposure to severe famine and subsequent risk of depressive symptoms in late adulthood: the China Health and Retirement Longitudinal Study," The British Journal of Psychiatry: the Journal of Mental Science, vol. 213, no. 4, pp. 579-586, 2018.

[77] P. He, L. Liu, J. M. I. Salas et al., "Prenatal malnutrition and adult cognitive impairment: a natural experiment from the 1959-1961 Chinese famine," The British Journal of Nutrition, vol. 120, no. 2, pp. 198-203, 2018.

[78] H. Rong, Y. Xi, Y. An et al., "The correlation between early stages of life exposed to Chinese famine and cognitive decline in adulthood: nutrition of adulthood plays an important role in the link?," Frontiers in Aging Neuroscience, vol. 9, p. 444, 2017.

[79] C. Wang, Y. An, H. Yu et al., "Association between exposure to the Chinese famine in different stages of early life and decline in cognitive functioning in adulthood," Frontiers in Behavioral Neuroscience, vol. 10, p. 146, 2016.

[80] J. Li, L. Na, H. Ma et al., "Multigenerational effects of parental prenatal exposure to famine on adult offspring cognitive function," Scientific Reports, vol. 5, no. 1, article 13792, 2015.

[81] S. He, J. Li, Z. Wang et al., "Early-life exposure to famine and late-life depression: does leukocyte telomere length mediate the association?," Journal of Affective Disorders, vol. 274, pp. 223-228, 2020.
[82] A. D. Stein, F. H. Pierik, G. H. Verrips, E. S. Susser, and L. H. Lumey, "Maternal exposure to the Dutch famine before conception and during Pregnancy," Epidemiology, vol. 20, no. 6, pp. 909-915, 2009.

[83] L. Zhang, J. L. Li, L. L. Zhang, L. L. Guo, H. Li, and D. Li, "Association and interaction analysis of body mass index and triglycerides level with blood pressure in elderly individuals in China," BioMed Research International, vol. 2018, Article ID 8934534, 31 pages, 2018.

[84] H. Sun, X. Ren, Z. Chen et al., "Association between body mass index and mortality in a prospective cohort of Chinese adults," Medicine (Baltimore), vol. 95, no. 32, article e4327, 2016.

[85] J. Hu, H. Xu, J. Zhu et al., "Association between body mass index and risk of cardiovascular disease-specific mortality among adults with hypertension in Shanghai, China," Aging, vol. 13, no. 5, pp. 6866-6877, 2021.

[86] N. H. Kim, J. Lee, T. J. Kim et al., "Body mass index and mortality in the general population and in subjects with chronic disease in Korea: a nationwide cohort study (2002-2010)," PLoS One, vol. 10, no. 10, article e0139924, 2015.

[87] K. Li, C. Yao, X. Yang et al., "Body mass index and the risk of cardiovascular and all-cause mortality among patients with hypertension: a population-based prospective cohort study among adults in Beijing, China," Journal of Epidemiology, vol. 26, no. 12, pp. 654-660, 2016.

[88] Q. N. Nguyen, S. T. Pham, V. L. Nguyen et al., "Time trends in blood pressure, body mass index and smoking in the Vietnamese population: a meta-analysis from multiple cross-sectional surveys," PLoS One, vol. 7, no. 8, article e42825, 2012.

[89] B. D. Power, H. Alfonso, L. Flicker, G. J. Hankey, B. B. Yeap, and O. P. Almeida, "Body adiposity in later life and the incidence of dementia: the health in men study," PLoS One, vol. 6, no. 3, article e17902, 2011.

[90] A. Ferra, M. del Mar Bibiloni, M. E. Zapata, J. Pich, A. Pons, and J. A. Tur, "Body mass index, life-style, and healthy status in free living elderly people in Menorca Island," The Journal of Nutrition, Health \& Aging, vol. 16, no. 4, pp. 298-305, 2012.

[91] C. S. Oxlund, M. Pareek, B. S. B. Rasmussen et al., "Body mass index, intensive blood pressure management, and cardiovascular events in the SPRINT trial," The American Journal of Medicine, vol. 132, no. 7, pp. 840-846, 2019.

[92] B. D. Cox, M. J. Whichelow, and A. T. Prevost, “The development of cardiovascular disease in relation to anthropometric indices and hypertension in British adults," International Journal of Obesity and Related Metabolic Disorders: Journal of the International Association for the Study of Obesity, vol. 22, no. 10, pp. 966-973, 1998.

[93] V. Atella, J. Kopinska, G. Medea et al., "Excess body weight increases the burden of age-associated chronic diseases and their associated health care expenditures," Aging, vol. 7, no. 10, pp. 882-892, 2015.

[94] S. P. Ramirez, W. McClellan, F. K. Port, and S. I. Hsu, "Risk factors for proteinuria in a large, multiracial, southeast Asian population," Journal of the American Society of Nephrology: JASN, vol. 13, no. 7, pp. 1907-1917, 2002.

[95] J. G. van Uffelen, J. Berecki-Gisolf, W. J. Brown, and A. J. Dobson, "What is a healthy body mass index for women in their seventies? Results from the Australian longitudinal study on women's Health," The Journals of Gerontology Series A: Biological Sciences and Medical Sciences, vol. 65A, no. 8, pp. 847-853, 2010. 
[96] W. Yang, J. P. Li, Y. Zhang et al., “Association between Body Mass Index and All-Cause Mortality in Hypertensive Adults: Results from the China Stroke Primary Prevention Trial (CSPPT)," Nutrients, vol. 8, no. 6, p. 384, 2016.

[97] L. M. Burgos, A. Gil Ramírez, L. Seoane et al., "Is the obesity paradox in cardiac surgery really a myth? Effect of body mass index on early and late clinical outcomes," Journal of Cardiothoracic and Vascular Anesthesia, vol. 35, no. 2, pp. 492-498, 2021.

[98] A. K. Dwivedi, P. Dubey, D. P. Cistola, and S. Y. Reddy, “Association between obesity and cardiovascular outcomes: updated evidence from meta-analysis studies," Current Cardiology Reports, vol. 22, no. 4, p. 25, 2020. 